\title{
TU/e EmonOWEN

\section{On the convergence of the eigencurrent expansion method applied to Linear Embedding via Green's Operators (LEGO)}

\section{Citation for published version (APA):}

Lancellotti, V., Hon, de, B. P., \& Tijhuis, A. G. (2010). On the convergence of the eigencurrent expansion method applied to Linear Embedding via Green's Operators (LEGO). IEEE Transactions on Antennas and Propagation, 58(10), 3231-3238. https://doi.org/10.1109/TAP.2010.2055804

DOI:

10.1109/TAP.2010.2055804

Document status and date:

Published: 01/01/2010

\section{Document Version:}

Publisher's PDF, also known as Version of Record (includes final page, issue and volume numbers)

\section{Please check the document version of this publication:}

- A submitted manuscript is the version of the article upon submission and before peer-review. There can be important differences between the submitted version and the official published version of record. People interested in the research are advised to contact the author for the final version of the publication, or visit the $\mathrm{DOI}$ to the publisher's website.

- The final author version and the galley proof are versions of the publication after peer review.

- The final published version features the final layout of the paper including the volume, issue and page numbers.

Link to publication

\section{General rights}

Copyright and moral rights for the publications made accessible in the public portal are retained by the authors and/or other copyright owners and it is a condition of accessing publications that users recognise and abide by the legal requirements associated with these rights.

- Users may download and print one copy of any publication from the public portal for the purpose of private study or research.

- You may not further distribute the material or use it for any profit-making activity or commercial gain

- You may freely distribute the URL identifying the publication in the public portal.

If the publication is distributed under the terms of Article 25fa of the Dutch Copyright Act, indicated by the "Taverne" license above, please follow below link for the End User Agreement:

www.tue.nl/taverne

Take down policy

If you believe that this document breaches copyright please contact us at:

openaccess@tue.nl

providing details and we will investigate your claim. 


\title{
On the Convergence of the Eigencurrent Expansion Method Applied to Linear Embedding via Green's Operators (LEGO)
}

\author{
Vito Lancellotti, Senior Member, IEEE, Bastiaan P. de Hon, and Anton G. Tijhuis, Member, IEEE
}

\begin{abstract}
The scattering from a large complex structure comprised of many objects may be efficiently tackled by embedding each object within a bounded domain (brick) which is described through a scattering operator. Upon electromagnetically combining the scattering operators we arrive at an equation which involves the total inverse scattering operator $S^{-1}$ of the structure: We call this procedure linear embedding via Green's operators (LEGO). To solve the relevant equation we then employ the eigencurrent expansion method (EEM) — essentially the method of moments with a set of basis and test functions that are approximations to the eigenfunctions of $S^{-1}$ (termed eigencurrents). We have investigated the convergence of the EEM applied to LEGO in cases when all the bricks are identical. Our findings lead us to formulate a simple and practical criterion for controlling the error of the computed solution a priori.
\end{abstract}

Index Terms-Boundary integral equations, composite structures, domain decomposition method, eigencurrent expansion method, equivalence principle, method of moments (MoM).

\section{INTRODUCTION}

$\mathbf{L}$ INEAR embedding via Green's operators (LEGO) is a domain decomposition method (DDM) particularly suited for solving the scattering and radiation of electromagnetic (EM) waves from (both 2-D and 3-D) large composite structures comprised of many bodies [1]-[4]. In the LEGO concept, similarly to other DDMs (e.g., [5]-[9]), we tear apart the structure into its constituent elements and we embed them within bounded (possibly interconnected) sub-domains, dubbed bricks. After describing each brick by means of a scattering operator, we capture the underlying physics of the whole original structure (i.e., the multiple scattering among the objects) by means of the total inverse scattering operator $S^{-1}$ [2], [4], which can be written analytically in a formal fashion.

The equation involving $\mathrm{S}^{-1}$ has to be solved for the (equivalent) scattered currents $q^{s}$ by a suitable numerical method. To this purpose, a direct application of the method of moments

Manuscript received November 13, 2009; revised March 24, 2010; accepted April 09, 2010. Date of publication July 01, 2010; date of current version October 06, 2010. This work was supported by the Postdoctoral Fund TU/e project no. 36/363450 and performed in the framework of the MEMPHIS project (http:// www.smartmix-memphis.nl).

The authors are with the Department of Electrical Engineering, Technical University of Eindhoven, 5600 MB Eindhoven, The Netherlands (e-mail: v.lancellotti@tue.nl; b.p.d.hon@tue.nl; a.g.tijhuis@tue.nl).

Color versions of one or more of the figures in this paper are available online at http://ieeexplore.ieee.org.

Digital Object Identifier 10.1109/TAP.2010.2055804
(MoM) [10] with some kind of sub-domain basis functions, such as the Rao-Wilton-Glisson (RWG) [11] div-conforming vector elements, may not be advisable when a large 3-D problem is addressed. In fact, the matrix of the resulting algebraic system becomes too big to be stored and formally inverted using a direct solver, even for moderate numbers of bricks.

In order to handle larger EM problems, in [2, Section IV], we proposed the eigencurrent expansion method (EEM), as an alternative to the bare MoM. In short, the EEM consists of using a set of approximated eigenfunctions of $\mathrm{S}^{-1}$ (termed eigencurrents) to represent $q^{s}$. To obtain the scattering operators of the bricks (and hence $\mathrm{S}^{-1}$ ) we actually resort to the MoM. Hence, the EEM is practically implemented as a basis change from the underlying set of RWG functions. In [2] we also demonstrated that the eigencurrents can be separated into two types, i.e., coupled and uncoupled. This observation enabled us to reduce the system matrix (in the basis of the eigencurrents) to block-diagonal form with just two blocks. The first one-associated with the coupled eigencurrents and fully populated-is ordinarily quite small as compared to the size of the whole matrix. Conversely, the other block-associated with the uncoupled eigencurrents and possibly huge - is diagonal and therefore it is effortlessly stored and (formally) inverted.

No clear transition exists between the two types of eigencurrents: Hence one may expect the computed solution to depend on the relative dimension of the sets of coupled and uncoupled eigencurrents. Concerning this, we numerically demonstrated in [2, Section V] that the EEM does converge, when the number of coupled eigencurrents is gradually increased (at the expense of the number of uncoupled eigencurrents). We also argued that a rule for dividing the eigencurrents is hard to state, for many factors come into play.

However, in an attempt to shed light on the convergence properties of the EEM applied to LEGO, we have worked out two purposeful case studies in the notable instance when the structure involves identical objects. Upon analyzing the eigenvalues of the scattering operator, we are indeed able to formulate a quite general criterion for determining the number of coupled eigencurrents a priori for a desired level of approximation.

To be more specific, in Section III we first investigate how the size and the shape and the EM properties of the body enclosed in a brick affect the spectrum of the relevant scattering operator. Secondly, we relate the error committed on the scattered currents $q^{s}$ (calculated through the EEM) to the eigenvalues of the scattering operators. It turns out that there is a simple relationship (practically linear) between the error and the 
eigenvalue pertinent to the highest-order coupled eigencurrent. What's more, this relationship appears virtually independent of the bricks' content and only weakly dependent on the frequency and the distance of the LEGO bricks.

Then, in Section IV we discuss our findings and draw up a practical rule for estimating the error of the computed solution or, conversely, for choosing the number of coupled eigencurrents required to attain a desired level of accuracy.

We mention that a convergence study was conducted for the synthetic-functions approach [7]. However, [7] does not provide an a-priori criterion for truncating the basis of the synthetic functions. To the best of our knowledge, the quest for a criterion for controlling the error has not been attempted so far for other DDMs.

\section{OVERVIEW OF LEGO/EEM AND NOTATION}

In what follows we conform to the assumptions and definitions adopted in our previous work on LEGO and EEM [2]. To reiterate briefly, we deal with a 3-D structure comprised of $N_{D}$ PEC or penetrable objects (immersed in a homogeneous background medium) which we embed in as many LEGO bricks $\mathcal{D}_{k}$, $k=1, \ldots, N_{D}$.

We employ the MoM to compute the scattering operator $S_{k k}$ of $\mathcal{D}_{k}$ and the transfer operator $\mathrm{T}_{k n}$ from $\partial \mathcal{D}_{n}^{-}$to $\partial \mathcal{D}_{k}^{+}, n=$ $1, \ldots, N_{D}$. To this purpose, we model both the surface $\mathcal{S}_{O}$ of the object inside $\mathcal{D}_{k}$ and the boundary $\partial \mathcal{D}_{k}$ with 3 -D surface triangular meshes on which we define $N_{O}$ and $2 N_{F}$ RWG functions, respectively. The algebraic counterparts of $S_{k k}$ and $\mathrm{T}_{k n}$ are then [2, Section IV]

$$
\begin{aligned}
& {\left[S_{k k}\right]=-\left[P_{k k}^{s}\right]^{-1}\left[P_{k o}\right]\left[X_{o o}\right]^{-1}\left[P_{o k}\right]} \\
& {\left[T_{k n}\right]=\left[P_{k k}^{i}\right]^{-1}\left[P_{k n}\right]}
\end{aligned}
$$

where the relevant operators are listed in [2, Tables I-III].

Finally, the algebraic equation to be solved is:

$$
[S]^{-1}\left[q^{s}\right]=\left[q^{i}\right]
$$

where the system matrix has rank $2 N_{F} N_{D}$. The diagonal blocks of $[S]^{-1}$ are the (formal) inverse of (1), whilst the off-diagonal blocks are the negative of (2). The column vectors $\left[q^{s}\right],\left[q^{i}\right]$ contain the expansion coefficient of the scattered and incident current densities defined on either side of $\partial \mathcal{D}_{k}$, viz.,

$$
\begin{aligned}
\left(\left[q^{s, i}\right]\right)_{k} & =\left[q_{k}^{s, i}\right] \\
\left(\left[q_{k}^{s, i}\right]\right)_{p} & = \begin{cases}J_{k p}^{s, i}, & p \in\left[1, N_{F}\right] \\
-M_{k p}^{s, i}, & p \in\left[N_{F}+1,2 N_{F}\right]\end{cases}
\end{aligned}
$$

where a normalization factor $\sqrt{\eta}(1 / \sqrt{\eta})$ is included in $J_{k p}^{s, i}\left(M_{k p}^{s, i}\right)$, with $\eta=\sqrt{\mu / \varepsilon}$ being the intrinsic impedance of the background medium.

To solve (3) with the EEM, we form a basis $U=\left\{\left[u_{p}^{(k)}\right]\right\}$ of approximated eigencurrents of $[S]^{-1}$ by juxtaposing the eigenvectors $\left[v_{p}^{(k)}\right]$ of $\left[S_{k k}\right][2]$. Thereby, $\left[u_{p}^{(k)}\right]$ vanishes everywhere but on the $k$ th brick, and two distinct eigencurrents with $k \neq n$ are orthogonal with respect to the standard inner product. Moreover, it is worth noticing that the scattering matrix $\left[S_{k k}\right]$ is rank-deficient whenever $2 N_{F}>N_{O}$, as the MoM matrix $\left[X_{o o}\right]$ in (1) has size $N_{O} \times N_{O}$. We will go back to this point in Section IV-A. For the time being, we solely observe that the number of coupled eigencurrents $N_{C}$, "contributed" by a brick, cannot exceed $\min \left\{2 N_{F}, N_{O}\right\}$ or, stated another way, the eigencurrents corresponding to the null space of $\left[S_{k k}\right]$, if they exist, are uncoupled.

Keeping this in mind, it is evident that inverting (3) through the EEM and as many coupled eigencurrents as possible is equivalent to solving (3) with the original basis of $2 N_{F} N_{D}$ RWG functions ${ }^{1}$. In fact, in this instance the EEM boils down to a mere basis change with no order reduction (see [2, Section IV]). For this reason we assume the latter solution as the reference for our comparisons, and we denote it by $\left[q_{\mathrm{ref}}^{s}\right]$.

Then, as a measure of the global accuracy of the solution computed with the EEM, we define the relative error on the scattered current coefficients as (cf. [7, Eq. (33)])

$$
\delta_{q^{s}} \stackrel{\text { def }}{=} \frac{\left\|\left[q^{s}\right]-\left[q_{\text {ref }}^{s}\right]\right\|_{2}}{\left\|\left[q_{\text {ref }}^{s}\right]\right\|_{2}}
$$

where $\|\cdot\|_{2}$ denote the vector 2-norm [13] in the space spanned by the rows of $[S]^{-1}$. To assess the pointwise accuracy of $q^{s}$, we introduce the local deviation with respect to the reference solution (cf. [7, Eq. (34)]), namely,

$$
\epsilon_{p}^{(k)} \stackrel{\text { def }}{=} \frac{\left|\left(\left[q_{k}^{s}\right]\right)_{p}-\left(\left[q_{k, \text { ref }}^{s}\right]\right)_{p}\right|}{\left\|\left[q_{\mathrm{ref}}^{s}\right]\right\|_{2}}
$$

where $\left(\left[q_{k}^{s}\right]\right)_{p}$ (and analogously $\left.\left(\left[q_{k, \text { ref }}^{s}\right]\right)_{p}\right)$ are defined in (4).

\section{Two CASE STUDIES}

The structures we considered are: A) an aggregate of $N_{D}=4$ perfect electric conducting (PEC) cubes and B) an aggregate of $N_{D}=3$ penetrable cylinders; in both cases the incident field was the plane wave $\boldsymbol{E}^{i}=\hat{\boldsymbol{x}} \exp (-\boldsymbol{j} \boldsymbol{k} \cdot \boldsymbol{r})[\mathrm{V} / \mathrm{m}]$ where $\boldsymbol{k}=$ $-2 \pi \hat{z} / \lambda_{0}$ with $\lambda_{0}$ the wavelength in vacuum.

Below we first describe the case studies. The discussion is postponed to Section IV for the sake of clarity.

\section{A. Scattering From PEC Cubes With Varying Size}

The PEC cubes (edge $a$ ) are arranged in a regular rectangular pattern (lattice constant $w$ ) parallel to the $x O y$ plane. In accordance with LEGO, we enclosed the cubes within $N_{D}=4$ touching cubic bricks (edge $d$ ). We considered five realizations of the cubes with increasing edge length, namely, $a / d \in\{0.3,0.4,0.5,0.6,0.7\}$. Additionally, we allowed for three different electrical sizes of the bricks, viz., $d / \lambda_{0} \in\{0.33357,0.66713,1.0007\}$.

For the sake of clarity, in Fig. 1 we report the case $a / d=0.7$ as well as the corresponding LEGO bricks. Also shown are

${ }^{1}$ To verify our assumption, for the 4-cube case study discussed in Section III-A we have computed the scattered current coefficients through the baseline MoM as follows. By solving an EFIE [12] over the cubes (with $4 N_{O}$ RWG functions) we have obtained the current density coefficients $\left[q_{o}\right]$. Hence, we have computed $\left[q_{k, \mathrm{MoM}}^{s}\right]=\left[P_{k k}^{s}\right]^{-1}\left[P_{k o}\right]\left(\left[q_{o}\right]\right)_{k}$, with $2 N_{F}$ functions over each brick's surface. The local deviation [defined similarly to (6)] between the LEGO/EEM solution with $N_{C}=\min \left\{2 N_{F}, N_{O}\right\}$ and $\left[q_{k, \text { MoM }}^{s}\right]$ results no larger than $10^{-3}$ in all of the instances we considered. 


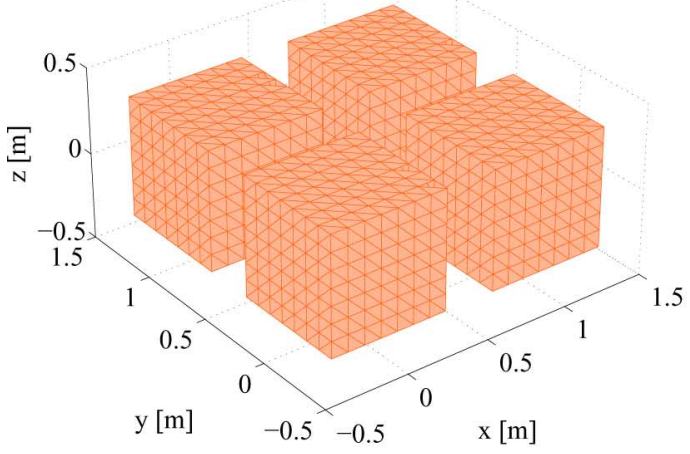

(a)

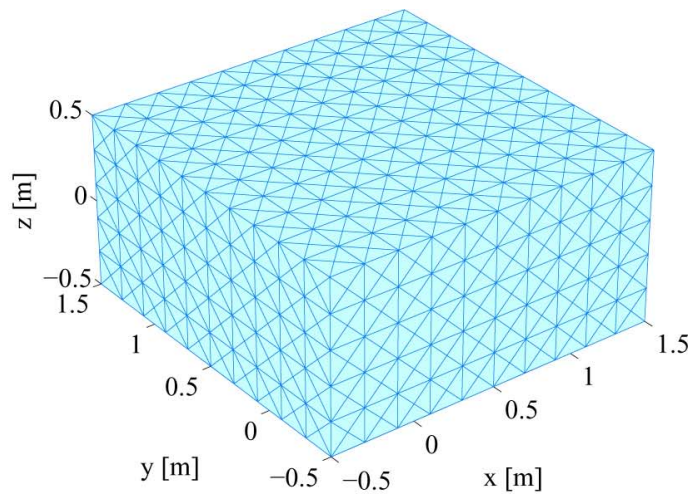

(b)

Fig. 1. Case study: (left) four PEC cubes arranged in a rectangular lattice (the realization $a / d=0.7$ is shown) and (right) LEGO model with as many cubic bricks each one enclosing a cube.

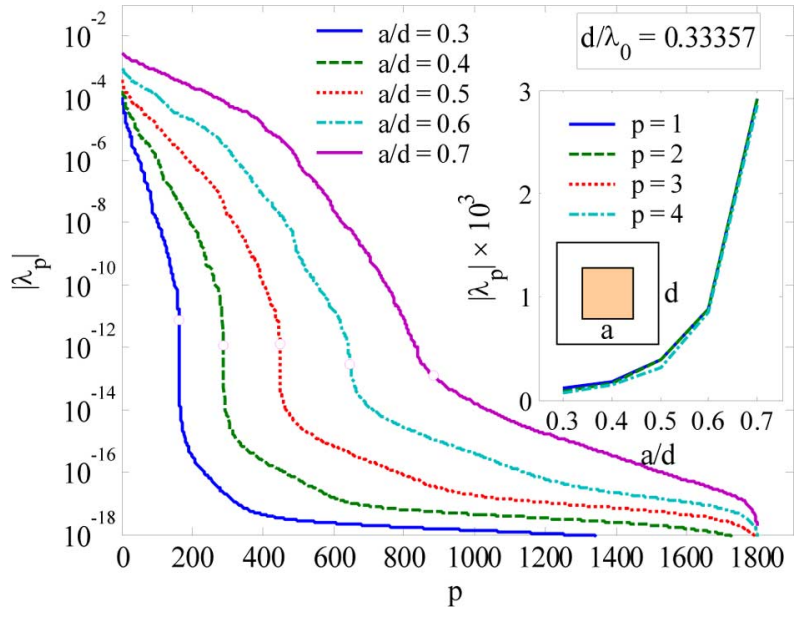

Fig. 2. Eigenvalues of $\left[S_{k k}\right]$ pertinent to the four cubic bricks shown in Fig. 1 as a function of the index $p$ for $d / \lambda_{0}=0.33357$ and for increasing values of $a / d$. The circles (o) mark the eigenvalues whose index $p=N_{O} \leq 2 N_{F}$. Inset: first four eigenvalues of $\left[S_{k k}\right]$ vs. $a / d$ and cartoon of a cube inside its LEGO brick.

the 3-D surface triangular meshes that are support to the underlying set of RWG basis functions employed in conjunction with MoM. In order to keep the mesh density at a constant value, over each cube's surface we adjusted the number of facets while increasing $a / d$. Consequently, we set $N_{O}$ to $\{162,288,450,648,882\}$, whereas the number of RWG functions on $\partial \mathcal{D}_{k}$ was fixed at $2 N_{F}=1800$. With these choices the average (electric) length of the edges in the mesh on $\partial \mathcal{D}_{k}$ and $\mathcal{S}_{O}$ is $l_{e} / \lambda_{0} \in\{0.053,0.107,0.161\}$ and $l_{e} / \lambda_{0} \in\{0.038,0.076,0.113\}$, respectively.

To begin with, we obtained the scattering operators $\left[S_{k k}\right](1)$ through the MoM: As a matter of fact, since the bricks are identical, we had to do the calculations for just one brick. Then, for each pair $\left(a / d, d / \lambda_{0}\right)$ we computed the eigenvalues $\lambda_{p}$ of $\left[S_{k k}\right]$. The results (i.e., $\left.\left|\lambda_{p}\right|\right)$ are plotted in Figs. 2-4 as a function of the eigenvalue index $p$ and for the three values of $d / \lambda_{0}$. The parameter of the lines is the ratio $a / d$, whilst the insets show the first four eigenvalues versus $a / d$. Finally, the circle on each curve tags the eigenvalue $\lambda_{N_{O}}$.

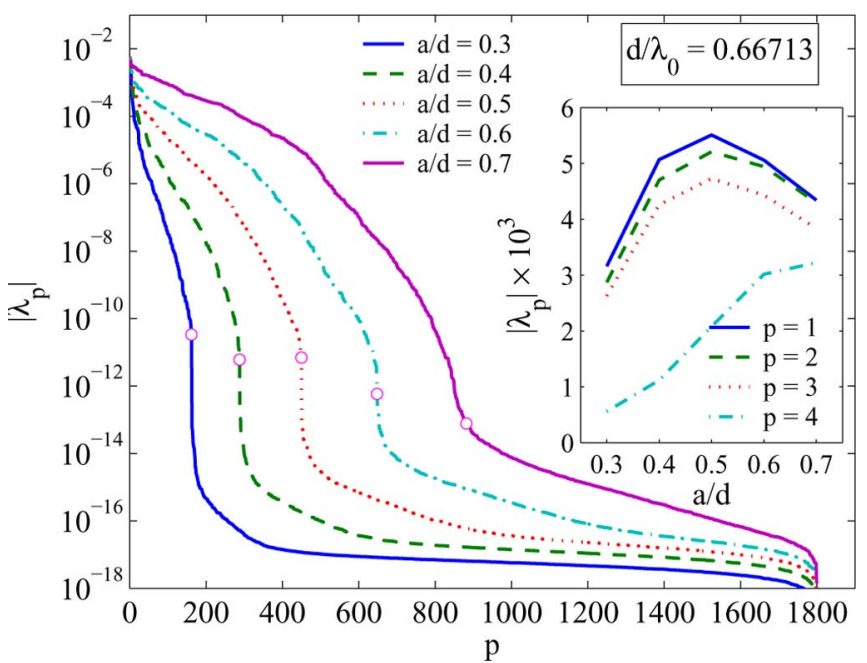

Fig. 3. Same as Fig. 2 for $d / \lambda_{0}=0.66713$.

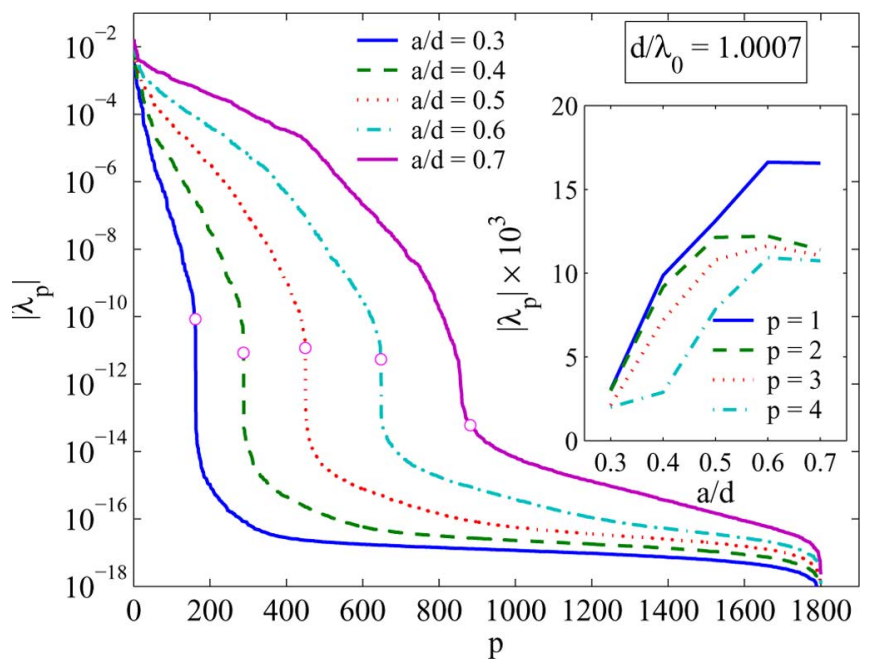

Fig. 4. Same as Fig. 2 for $d / \lambda_{0}=1.0007$.

Secondly, we repeatedly solved (3) with the EEM, while retaining an increasingly larger number of coupled eigencurrents $N_{C}$ per brick. To be specific, $N_{C}$ was set upon 

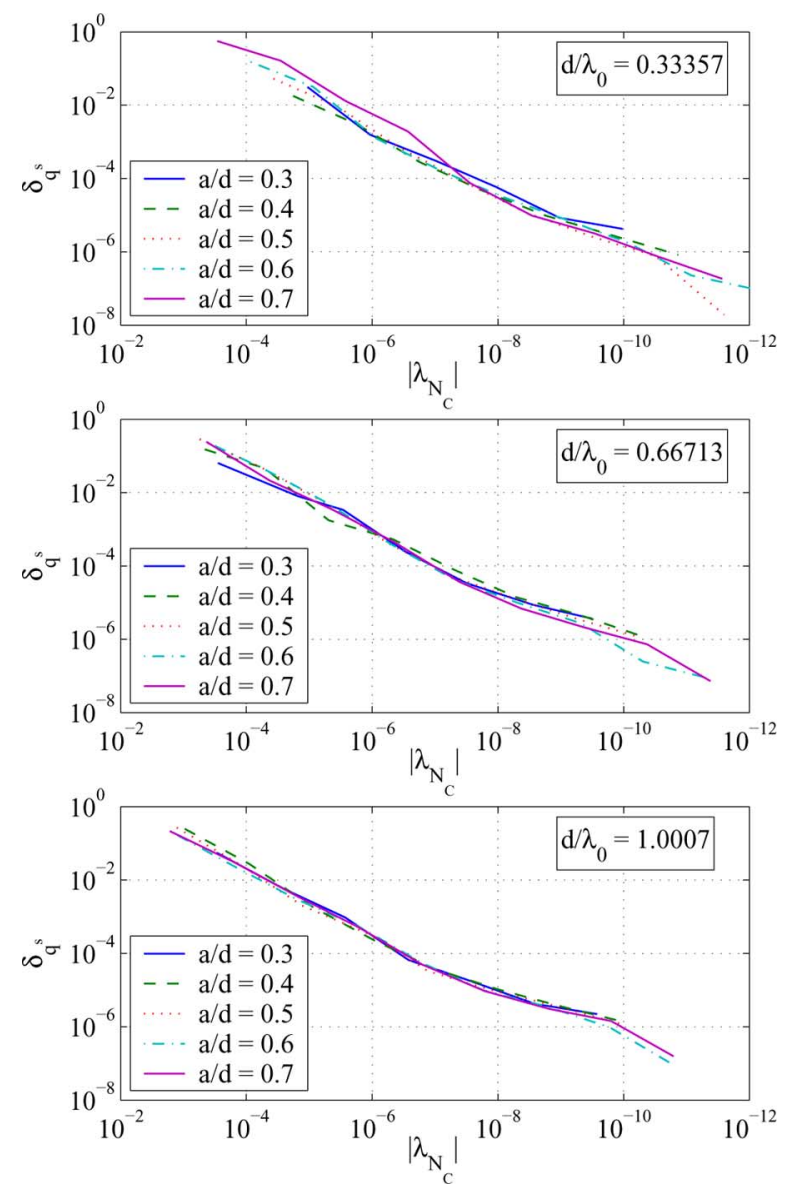

Fig. 5. 2-norm error $\delta_{q}$ of the equivalent scattered current coefficients $\left[q^{s}\right]$ relevant to the four cubic bricks shown in Fig. 1 as a function of the eigenvalue pertinent to the last coupled eigencurrent and for increasing values of $a / d$.

TABLE I

$N_{C}$ FOR THE 4-Cube CASE STUdY AND $d / \lambda_{0}=0.33357$

\begin{tabular}{cccccc}
\hline & \multicolumn{5}{c}{$a / d$} \\
\cline { 2 - 6 } $10^{-t}$ & 0.3 & 0.4 & 0.5 & 0.6 & 0.7 \\
\hline $10^{-1}$ & 18 & 34 & 58 & 112 & 185 \\
$10^{-2}$ & 48 & 91 & 142 & 247 & 368 \\
$10^{-3}$ & 80 & 133 & 228 & 331 & 474 \\
$10^{-4}$ & 101 & 181 & 289 & 398 & 537 \\
$10^{-5}$ & 130 & 228 & 340 & 468 & 598 \\
$10^{-6}$ & 150 & 260 & 388 & 503 & 669 \\
$10^{-7}$ & 161 & 282 & 420 & 559 & 724 \\
$10^{-8}$ & 162 & 287 & 446 & 614 & 772 \\
$10^{-9}$ & 162 & 288 & 450 & 641 & 814 \\
$10^{-10}$ & 162 & 288 & 450 & 648 & 854 \\
\hline
\end{tabular}

counting all the eigencurrents whose eigenvalues satisfy $\left|\lambda_{p}\right| \geq\left|\lambda_{N_{C}}\right|=10^{-t}\left|\lambda_{1}\right|, t=1, \ldots, 10$ : The relevant values (versus the threshold $10^{-t}$ and $a / d$ ) are listed in Tables I-III. Afterwards, from the knowledge of $\left[q^{s}\right]$ we calculated the corresponding 2-norm error with (5). In doing so, we obtained the sets of curves plotted in Fig. 5 for the three values of $d / \lambda_{0}$. The quantity on the horizontal axis (note the reversed scale) is $\left|\lambda_{N_{C}}\right|$, i.e., the magnitude of the eigenvalue corresponding to the last coupled eigencurrent contributed by $\left[S_{k k}\right]$. Once again the parameter of the lines is $a / d$.

Thirdly, to probe the effect of the lattice constant, we repeated the test above for $a / d=0.5, d / \lambda_{0}=1.0007$ and $w / d \in$
TABLE II

$N_{C}$ FOR THE 4-CUBE CASE STUDY AND $d / \lambda_{0}=0.66713$

\begin{tabular}{cccccc}
\hline & \multicolumn{5}{c}{$a / d$} \\
\cline { 2 - 6 } $10^{-t}$ & 0.3 & 0.4 & 0.5 & 0.6 & 0.7 \\
\hline $10^{-1}$ & 6 & 8 & 11 & 40 & 150 \\
$10^{-2}$ & 23 & 31 & 69 & 157 & 353 \\
$10^{-3}$ & 39 & 75 & 152 & 292 & 478 \\
$10^{-4}$ & 74 & 125 & 238 & 377 & 546 \\
$10^{-5}$ & 100 & 182 & 299 & 446 & 622 \\
$10^{-6}$ & 127 & 219 & 350 & 500 & 697 \\
$10^{-7}$ & 147 & 254 & 391 & 556 & 758 \\
$10^{-8}$ & 162 & 280 & 433 & 609 & 800 \\
$10^{-9}$ & 162 & 288 & 450 & 643 & 836 \\
$10^{-10}$ & 162 & 288 & 450 & 648 & 855
\end{tabular}

TABLE III

$N_{C}$ FOR THE 4-CuBE CASE STUDY AND $d / \lambda_{0}=1.0007$

\begin{tabular}{cccccc}
\hline & \multicolumn{5}{c}{$a / d$} \\
\cline { 2 - 6 } $10^{-t}$ & 0.3 & 0.4 & 0.5 & 0.6 & 0.7 \\
\hline $10^{-1}$ & 14 & 12 & 17 & 21 & 77 \\
$10^{-2}$ & 34 & 38 & 67 & 122 & 273 \\
$10^{-3}$ & 54 & 75 & 151 & 253 & 452 \\
$10^{-4}$ & 86 & 130 & 228 & 361 & 527 \\
$10^{-5}$ & 109 & 182 & 290 & 431 & 609 \\
$10^{-6}$ & 133 & 219 & 349 & 492 & 692 \\
$10^{-7}$ & 155 & 255 & 394 & 557 & 760 \\
$10^{-8}$ & 162 & 279 & 436 & 611 & 800 \\
$10^{-9}$ & 162 & 287 & 449 & 644 & 835 \\
$10^{-10}$ & 162 & 288 & 450 & 648 & 855
\end{tabular}

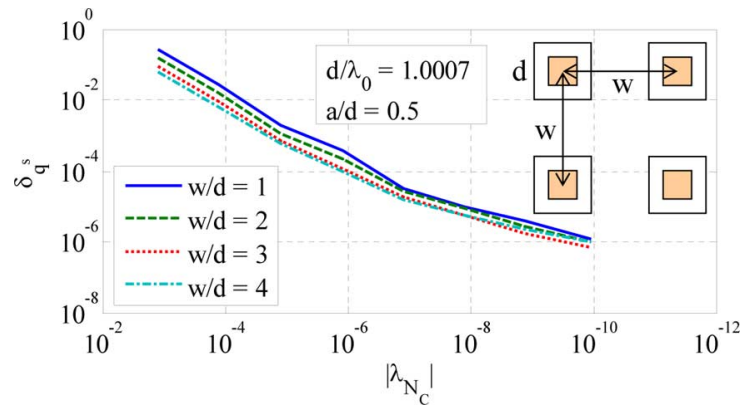

Fig. 6. 2-norm error $\delta_{q}$ of the equivalent scattered current coefficients $\left[q^{s}\right]$ relevant to the four cubic bricks shown in Fig. 1 as a function of the eigenvalue pertinent to the last coupled eigencurrent and for increasing values of $w / d$. Inset: cartoon of the cubic bricks and geometrical quantities.

$\{1,2,3,4\}$, and we calculated $\delta_{q^{s}}$. The selected values of $N_{C}$ can be retrieved from Table III as well. The results are plotted in Fig. 6 versus $\left|\lambda_{N_{C}}\right|$, where the parameter is now $w / d$.

Eventually, for all of the cases above we calculated the local deviation of $q^{s}$ from (6). As an example, in Fig. 7 we report $\epsilon_{p}^{(k)}$ for the realization $a / d=0.4, d / \lambda_{0}=1.0007$ and $\left|\lambda_{N_{C}}\right| \approx$ $10^{-2}\left|\lambda_{1}\right|$-which corresponds to $N_{C}=38$ and $N_{C} N_{D}=$ 152. It can be noted that the largest value taken on by $\epsilon_{p}^{(k)}$ is $\approx 1.410^{-3}$ and that the accuracy of the scattered current densities $\boldsymbol{J}_{k}^{s}$ and $\boldsymbol{M}_{k}^{s}$ [2] is basically the same.

\section{B. Scattering From Dielectric Cylinders With Varying Permittivity}

The dielectric cylinders (radius $a$ and height $h$ ) are arranged in a regular triangular pattern parallel to the $x O y$ plane. We embedded the cylinders within $N_{D}=3$ bricks which are hexagonal right prisms (edge $l$ and height $H$.) To investigate the effect 


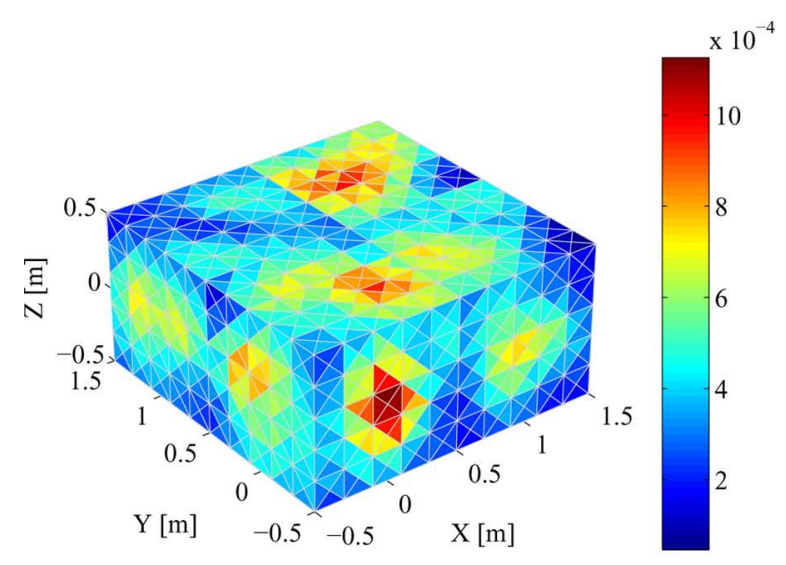

(a)

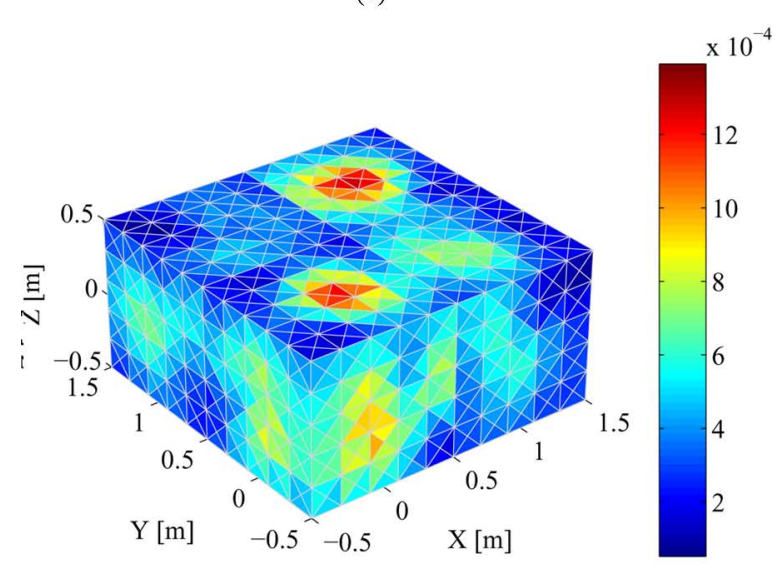

(b)

Fig. 7. Local deviation from the reference solution of (top) normalized $\boldsymbol{J}_{k}^{s}$ and (bottom) normalized $\boldsymbol{M}_{k}^{s}$ relevant to the four cubic bricks shown in Fig. 1 for the case $a / d=0.4, d / \lambda_{0}=1.0007$ and $\left|\lambda_{N_{C}}\right| \approx 10^{-2}\left|\lambda_{1}\right|$.

of the material properties, we considered five different realizations of the cylinders with different relative permittivity, namely, $\varepsilon_{r} \in\{5,10,15,20,25\}$. Instead, we held the relative size of cylinders and bricks fixed $(a / l=0.4, H / h=15 / 11, a / h=$ $4 / 11)$ and the brick's size constant $\left(l / \lambda_{0}=0.13343\right.$.) Shown in Fig. 8 are the cylinders and the bricks along with their 3-D surface triangular patching. In particular, the number of RWG functions defined on a brick's boundary and on a cylinder's surface is $2 N_{F}=2160$ and $N_{O}=2 \times 756=1512$. Accordingly, the average (electric) length of the edges in the mesh is $l_{e} / \lambda_{0}=0.036$ on $\partial \mathcal{D}_{k}$ and $l_{e} \sqrt{\varepsilon_{r}} / \lambda_{0} \in\{0.034,0.048,0.059,0.068,0.077\}$ on $\mathcal{S}_{O}$.

As in the previous case study, we computed the scattering operator of one brick. Calculation of the induced current densities on the embedded cylinder was carried out through the PMCHWT set of integral equations [12]. The spectrum of $\left[S_{k k}\right]$ is plotted in Fig. 9 versus $p$; the parameter of the curves is $\varepsilon_{r}$, whereas the inset displays the first four eigenvalues as a function of $\varepsilon_{r}$.

Upon repeatedly inverting (3) by applying the EEM with more and more coupled eigencurrents $N_{C}$ (see Table IV for the relevant values), we computed $\left[q^{s}\right]$ and subsequently $\delta_{q^{s}}$ from (5). The results are presented in Fig. 10 as a function of $\left|\lambda_{N_{C}}\right|$ for $l / \lambda_{0}=0.13343$ and the five values of permittivity selected above.

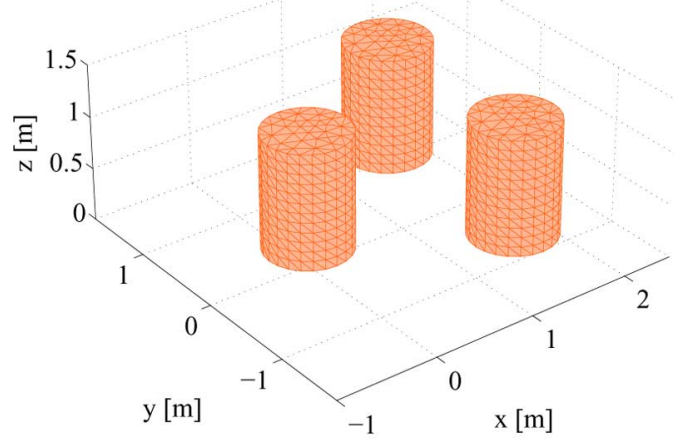

(a)

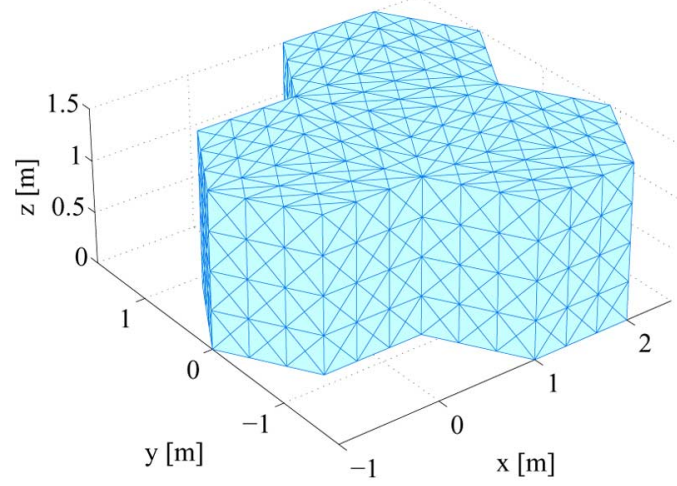

(b)

Fig. 8. Case study: (top) three dielectric cylinders arranged in a triangular lattice and (bottom) LEGO model with as many hexagonal bricks each one enclosing a cylinder.

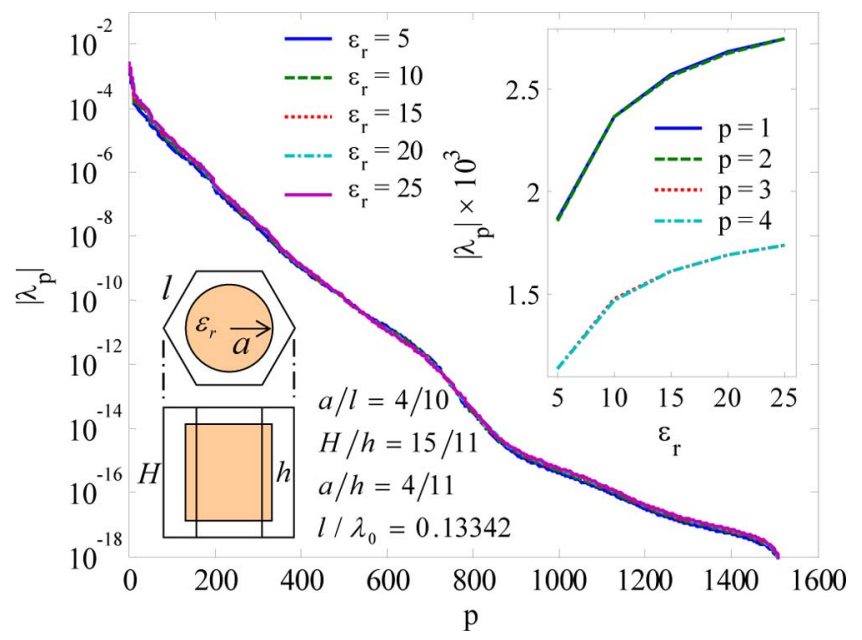

Fig. 9. Normalized eigenvalues of $\left[S_{k k}\right]$ pertinent to the three hexagonal bricks shown in Fig. 8 as a function of the index $p$ for increasing values of $\varepsilon_{r}$. Insets: first four eigenvalues of $\left[S_{k k}\right]$ vs. $\varepsilon_{r}$ and cartoon of a cylinder inside its LEGO brick.

\section{DISCUSSION OF RESULTS}

\section{A. Spectrum of $\left[S_{k k}\right]$}

From Figs. 2-4 and 9 we observe the following.

1) The decay rate of the spectrum of $\left[S_{k k}\right]$ increases as the relative size $(a / d)$ of the body enclosed in $\mathcal{D}_{k}$ is reduced 


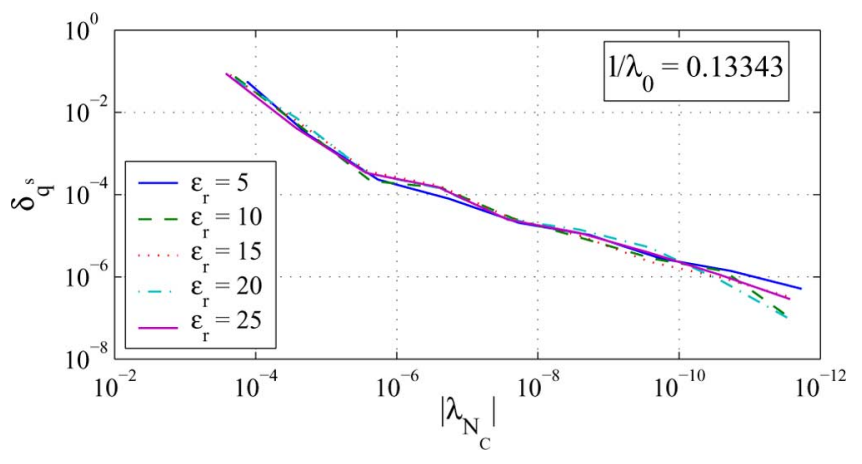

Fig. 10. 2-norm error $\delta_{q}$ of the equivalent scattered current coefficients $\left[q^{s}\right]$ relevant to the three hexagonal bricks shown in Fig. 8 as a function of the eigenvalue pertinent to the last coupled eigencurrent for $l / \lambda_{0}=0.13343$ and increasing values of $\varepsilon_{r}$.

TABLE IV

$N_{C}$ FOR THE 3-CyLINDER CASE STUdY

\begin{tabular}{cccccc}
\hline & \multicolumn{5}{c}{$\varepsilon_{r}$} \\
\cline { 2 - 6 } $10^{-t}$ & 5 & 10 & 15 & 20 & 25 \\
\hline $10^{-1}$ & 13 & 13 & 13 & 14 & 15 \\
$10^{-2}$ & 67 & 69 & 69 & 69 & 72 \\
$10^{-3}$ & 145 & 145 & 143 & 145 & 147 \\
$10^{-4}$ & 217 & 214 & 214 & 213 & 215 \\
$10^{-5}$ & 298 & 296 & 293 & 297 & 297 \\
$10^{-6}$ & 377 & 373 & 373 & 372 & 373 \\
$10^{-7}$ & 478 & 470 & 467 & 465 & 465 \\
$10^{-8}$ & 588 & 571 & 565 & 562 & 561 \\
$10^{-9}$ & 695 & 679 & 677 & 670 & 667 \\
$10^{-10}$ & 754 & 751 & 748 & 747 & 749 \\
\hline
\end{tabular}

or, from another perspective, the boundary of the brick is moved farther away from the surface of the object.

2) The bigger the object with respect to the enclosing brick, the larger the amplitude of the $p$ th eigenvalue, $\left|\lambda_{p}\right|$. Apparently, this applies to most of the spectrum, but it may not be true for the very first eigenvalues (as confirmed by the inset in Fig. 3) which correspond to the strongest reflections in view of the physical meaning of $\left[S_{k k}\right]$.

It is worthwhile noticing at this point that, as long as the medium embedded within a brick is passive, the eigenvalues of $\left[S_{k k}\right]$ cannot exceed unity (in magnitude), based on physical grounds. Numerical experiments (not reported here) show that the $\left|\lambda_{p}\right|$ 's do approach unity when the enclosed object fills the brick almost totally.

3) The spectrum of $\left[S_{k k}\right]$ depends only weakly on the EM properties of the embedded object, with the most evident variations regarding the first eigenvalues (inset of Fig. 9.)

4) The electric size of the bricks $\left(d / \lambda_{0}\right)$ does not seem to play a major role, at least in the present numerical experiments. At any rate, for a given geometry, increasing the frequency causes the whole spectrum to drift toward higher values of $\left|\lambda_{p}\right|$.

5) In cases when $2 N_{F}>N_{O}$, the spectrum of $\left[S_{k k}\right]$ exhibits an abrupt discontinuity occurring for $p=N_{O}$ : From that index on the eigenvalues are theoretically zero and in practice close to or below the threshold of numerical noise for double-precision floating-point operations. However, we emphasize that we are not in trouble with the calculation of $[S]^{-1}$, even though this entails $\left[S_{k k}\right]^{-1}$.
TABLE V

CONDITION Numbers of $\left[P_{k k}^{s}\right]$ AND $\left[X_{o o}\right]$ FOR THE 4-CUBE CASE STUdY

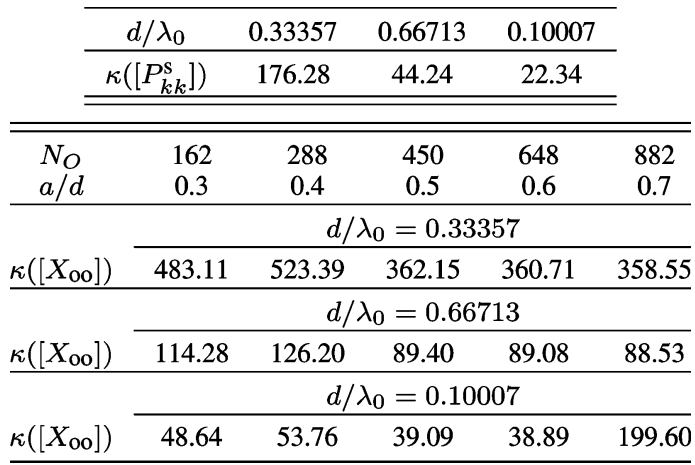

In fact, as detailed in [2, Section IV], on the one hand, we never compute $\left[S_{k k}\right]^{-1}$ as such, but to apply the EEM we just invert the $N_{C}$ eigenvalues corresponding to the coupled eigencurrents, whereas, on the other hand, we need not invert the eigenvalues of the uncoupled eigencurrents (which may be null.) Therefore the stability of the EEM is not endangered, provided $N_{C} \leq \min \left\{2 N_{F}, N_{O}\right\}$, as anticipated in Section II. Only, when $2 N_{F}>N_{O},[S]^{-1}$ has to be interpreted formally, because $\left[S_{k k}\right]^{-1}$ is meaningless.

One may also argue, in the light of Figs. 2-4 and 9, that the spectral condition number $\kappa$ of $\left[S_{k k}\right]$ is very high. In general, though, neither $\left[P_{k k}^{s}\right]$ nor $\left[X_{o o}\right]$ [see (1)] are to be blamed for the overall ill-conditioning of $\left[S_{k k}\right]$. To back up our statement, in Table $\mathrm{V}$ we list $\kappa\left(\left[P_{k k}^{s}\right]\right)$ and $\kappa\left(\left[X_{o o}\right]\right)$ for the 4-cube case study of Section III-A. In practice, $\left[P_{k k}^{s}\right]\left(\left[X_{o o}\right]\right)$ happens to be more or less well-conditioned depending on the kind of integral equations posed on $\partial \mathcal{D}_{k}\left(\mathcal{S}_{O}\right)$ and on the mesh density. Thereby, the inversion of $\left[P_{k k}^{s}\right]$ and $\left[X_{o o}\right]$ (and hence the calculation of $\left.\left[S_{k k}\right]\right)$ is not an issue.

As a matter of fact, $\kappa\left(\left[S_{k k}\right]\right)$ is mostly affected by the propagation matrices $\left[P_{o k}\right]$ and $\left[P_{k o}\right]$, which relate currents on $\partial \mathcal{D}_{k}$ to fields on $\mathcal{S}_{O}$ and viceversa [2, Table III]. These matrices are intrinsically rank-deficient in that the number of basis functions on $\partial \mathcal{D}_{k}\left(2 N_{F}\right)$ and $\mathcal{S}_{O}\left(N_{O}\right)$ need not be the same. Rather, $2 N_{F}$ and $N_{O}$ have to be adjusted independently, according to the commonly accepted meshing criterion (i.e., mesh edge $\approx 0.1 \times$ wavelength). Nevertheless, even though $2 N_{F}$ and $N_{O}$ coincided, the effective rank of $\left[P_{o k}\right]$ and $\left[P_{k o}\right]$ could be smaller than $2 N_{F}=N_{O}$, since the entries of $\left[P_{o k}\right]$ and $\left[P_{k o}\right]$ can be proven to decay to zero when the distance between $\partial \mathcal{D}_{k}$ and $\mathcal{S}_{O}$ is increased. Accordingly, the columns of $\left[P_{o k}\right]$ and $\left[P_{k o}\right]$ become almost linearly dependent, and a singular value decomposition reveals that the higher-order singular values of $\left[P_{o k}\right]$ and $\left[P_{k o}\right]$ are null or nearly so.

\section{B. Relative Error on the Computed Currents}

From Figs. 5, 6 and 10 we observe the following.

1) The relative error $\delta_{q^{s}}$ decreases linearly (in a logarithmic scale) with $\left|\lambda_{N_{C}}\right|$, viz., the amplitude of the eigenvalue pertaining to the highest-order coupled eigencurrent. Notice that the actual number of coupled eigencurrents $N_{C}$, relative to a given value of $\left|\lambda_{N_{C}}\right|$, may be different for different 
geometries and frequencies, as suggested by Figs. $2-4$ and 9.

2) The relative error $\delta_{q^{s}}$ appears virtually independent of the size and the EM properties of the objects and only weakly affected by the frequency. In practice, when $d / \lambda_{0}$ increases, $\delta_{q^{s}}$ diminishes, which shows as a leftward drift of the error curves.

$3)$ For given frequency and geometry, $\delta_{q^{s}}$ improves when the bricks are located farther away. This effect, however, may not be explained by the behavior of $\left|\lambda_{p}\right|$, since $\left[S_{k k}\right]$ is not affected by the relative position of the bricks in space. The latter, though, enters through the transfer operators $\left[T_{k n}\right]$ (2).

Based on established multipole expansion methods [14], [15], we expect the entries of $\left[T_{k n}\right]$ to become smaller and smaller when $\mathcal{D}_{k}$ and $\mathcal{D}_{n}$ are set farther and farther away. Consequently, the eigencurrents are more and more attenuated while propagating from $\partial \mathcal{D}_{n}^{-}$to $\partial \mathcal{D}_{k}^{+}$. This means that ever more eigencurrents become uncoupled, if $w / d$ is increased. Therefore, holding $\left|\lambda_{N_{C}}\right|$ (and hence $N_{C}$ ) fixed must yield more accurate results, as confirmed by Fig. 6 .

\section{Number of Coupled Eigencurrents}

The diagrams of Figs. 5, 6 and 10 can be used in two complementary ways. First of all, entering the plots with $\left|\lambda_{N_{C}}\right|$, we can determine the accuracy of the solution to (3) obtained through the EEM applied with $N_{C} N_{D}$ coupled eigencurrents. Alternatively, and perhaps more fruitfully, entering the plots with $\delta_{q^{s}}$, we can read off $\left|\lambda_{N_{C}}\right|$, whence we determine $N_{C}$, i.e., the number of coupled eigencurrents necessary for attaining the desired accuracy.

Now, since the error curves in Figs. 5, 6 and 10 are practically straight lines with slope $\approx 1$ (recall the horizontal scale is reversed), by direct inspection we are able to fit the pairs $\left(\left|\lambda_{N_{C}}\right|, \delta_{q^{s}}\right)$ by means of the mathematical linear mapping

$$
\log _{10} \delta_{q^{s}} \approx \log _{10}\left|\lambda_{N_{C}}\right|+\alpha\left(\lambda_{0}, \frac{a}{d}, \frac{w}{d}, \ldots\right)
$$

where $10^{\alpha}$ (which constitutes the vertical intercept) represents the value of $\delta_{q^{s}}$ when $\left|\lambda_{N_{C}}\right|=1$.

From the preceding discussion, it is clear that in (7) $\alpha$ ought to be determined specifically for the problem under consideration. Nonetheless, our findings also tell us that $\alpha$ is only weakly dependent on $\lambda_{0}, a / d, w / d$, and the EM properties of the embedded object. In the light of Figs. 5, 6 and 10, we conservatively set $\alpha \approx 3$, which should be fine for reasonable values of the relevant parameters. Thus, for the sake of argument, assuming we want to solve (3) with an error $\delta_{q^{s}} \approx 10^{-3}$, then from (7) we know that $\left|\lambda_{N_{C}}\right| \approx 10^{-6}$. From the knowledge of $\left|\lambda_{N_{C}}\right|$ we deduce $N_{C}$ by simply counting the eigenvalues that satisfy the condition $\left|\lambda_{p}\right| \geq\left|\lambda_{N_{C}}\right|$.

\section{CONCLUSIONS}

Upon investigating two case studies, we have addressed the convergence of the EEM applied to LEGO. In particular, by analyzing the effects that geometrical and EM parameters as well as the frequency have on the eigenvalues of a brick's scattering operator, we have empirically found that there exists a simple relationship between the error on the current and the amplitude of the eigenvalue corresponding to the highest-order coupled eigencurrent. We have translated this law into a compact approximate mathematical formula, which can be practically implemented in a numerical code for choosing the required number of coupled eigencurrents a priori.

\section{ACKNOWLEDGMENT}

The authors would like to thank the Reviewers for their constructive comments which helped improve the manuscript.

\section{REFERENCES}

[1] A. M. van de Water, B. P. de Hon, M. C. van Beurden, A. G. Tijhuis, and P. de Maagt, "Linear embedding via Green's operators: A modeling technique for finite electromagnetic band-gap structures," Phys. Rev. $E$, vol. 72, no. 5, pp. 1-11, Nov. 2005.

[2] V. Lancellotti, B. P. de Hon, and A. G. Tijhuis, "An eigencurrent approach to the analysis of electrically large 3-D structures using linear embedding via Green's operators," IEEE Trans. Antennas Propag., vol. 57, no. 11 , pp. 3575-3585, Nov. 2009.

[3] V. Lancellotti, B. P. de Hon, and A. G. Tijhuis, "A total inverse scattering operator formulation for solving large structures with LEGO," in Proc. Int. Conf. on Electromagnetics in Advanced Applications, ICEAA'09, Sep. 2009, pp. 335-338.

[4] V. Lancellotti, B. P. de Hon, and A. G. Tijhuis, "Sensitivity analysis of 3-D composite structures through linear embedding via Green's operators," Progr. Electromagn. Res., vol. 100, pp. 309-325, Jan. 2010.

[5] J. Yeo, V. V. S. Prakash, and R. Mittra, "Efficient analysis of a class of microstrip antennas using the characteristic basis function method (CBFM)," Microw. Opt. Technol. Lett., vol. 39, no. 6, pp. 456-464, 2003.

[6] M. K. Li and W. C. Chew, "Wave-field interaction with complex structures using equivalence principle algorithm," IEEE Trans. Antennas Propag., vol. 55, no. 1, pp. 130-138, Jan. 2007.

[7] L. Matekovitz, V. A. Laza, and G. Vecchi, "Analysis of large complex structures with the synthetic-functions approach," IEEE Trans. Antennas Propag., vol. 55, no. 9, pp. 2509-2521, Sep. 2007.

[8] P. Ylä-Oijala and M. Taskinen, "Electromagnetic scattering by large and complex structures with surface equivalence principle algorithm,' Waves Random Complex Media, vol. 19, no. 1, pp. 105-125, Feb. 2009.

[9] G. Xiao, J.-F. Mao, and B. Yuan, "A generalized surface integral equation formulation for analysis of complex electromagnetic systems," IEEE Trans. Antennas Propag., vol. 57, no. 3, pp. 701-710, Mar. 2009.

[10] R. F. Harrington, Field Computation by Moment Methods. New York: MacMillan, 1968.

[11] S. M. Rao, D. R. Wilton, and A. W. Glisson, "Electromagnetic scattering by surfaces of arbitrary shape," IEEE Trans. Antennas Propag., vol. 30, no. 3, pp. 409-418, May 1982.

[12] A. F. Peterson, S. L. Ray, and R. Mittra, Computational Methods for Electromagnetics. Piscataway, NJ: IEEE Press, 1998.

[13] G. H. Golub and C. F. van Loan, Matrix Computations. Baltimore, MD: Johns Hopkins Univ. Press, 1996.

[14] N. Engheta, W. D. Murphy, V. Rokhlin, and M. S. Vassiliou, "The fast multipole method (FMM) for electromagnetic problems," IEEE Trans. Antennas Propag., vol. 40, no. 7, pp. 634-641, Jun. 1992.

[15] J. D. Jackson, Classical Electrodynamics, 3rd ed. Chichester, U.K.: Wiley, 1999.

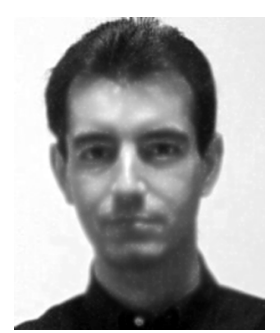

Vito Lancellotti (M'04-SM'09) was born in Torino, Italy, in 1968. He received the Laurea (M.Sc.) degree (with honors) in electrical engineering and the Ph.D. degree in electronics and communications both from Politecnico di Torino, Italy, in 1995 and 1999, respectively.

In early 1999, he joined the Telecom Italia Lab, Torino, as a Senior Researcher and was involved in projects concerning TCP/IP and ATM networks. In June 2000, he became Senior Researcher at the Milan-based subsidiary of Corning (now Avanex), where he worked on broadband electro-optic lithium niobate modulators and optical waveguides. From 2002 to 2008, he served as a Research Fellow and Lecturer in the Department of Electrical Engineering, Politecnico di Torino, where he substantially contributed to the development of the TOPICA code, 
devised for analysis and design of plasma facing antennas for magnetically controlled nuclear fusion. In 2005, he was appointed Visiting Scientist at Massachusetts Institute of Technology, Cambridge, and in 2007 conducted research at the Max-Planck-Institut fur Plasmaphysik, Garching, Germany. As of April 2008, he has been a Research Fellow and Lecturer with the Faculty of Electrical Engineering, Eindhoven University of Technology, Eindhoven, The Netherlands. His current research interests mainly concern efficient techniques for the computational modeling of electromagnetic fields in large structures.

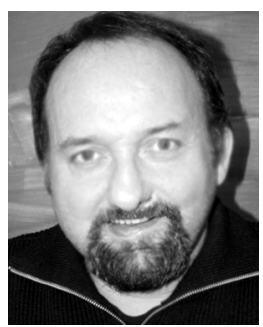

Bastiaan P. de Hon was born in 1966 in Amstelveen, The Netherlands. He received the M.Sc. and Ph.D. degrees (both cum laude) in electrical engineering from the Delft University of Technology, Delft, The Netherlands, in 1991 and 1996, respectively.

Since 1996, he has been with the Electromagnetics Group, Department of Electrical Engineering, Eindhoven University of Technology, Eindhoven, The Netherlands. From 1996 until 2000, he was on a fellowship awarded by the Royal Netherlands Academy of Arts and Sciences and, from 2000, he was a Lecturer. He has been a summer student at CERN, Geneva (Switzerland) and at Schlumberger Cambridge Research, Cambridge, U.K., and a Visiting Scientist at the University of Tel Aviv, Tel Aviv, Israel, and the University of Glasgow, UK. His research interests include theoretical and numerical aspects of electromagnetic, acoustic and elastic wave phenomena.
Dr. de Hon received the Steven Hoogendijk Award in 1998 for his Ph.D. thesis.

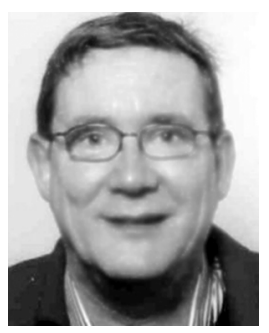

Anton G. Tijhuis ( $\mathrm{M}^{\prime} 88$ ) was born in Oosterhout N.B., The Netherlands, in 1952. He received the M.Sc. degree in theoretical physics from Utrecht University, Utrecht, The Netherlands, in 1976, and the Ph.D. degree (cum laude) from the Delft University of Technology, Delft, The Netherlands, in 1987.

From 1976 to 1986 and 1986 to 1993 , he was an Assistant and Associate Professor with the Laboratory of Electromagnetic Research, Faculty of Electrical Engineering, Delft University of Technology. In 1993, he became a Full Professor of electromagnetics with the Faculty of Electrical Engineering, Eindhoven University of Technology, Eindhoven, The Netherlands. He has been a Visiting Scientist with the University of Colorado at Boulder, the University of Granada, Granada, Spain, the University of Tel Aviv, Tel Aviv, Israel, and with McDonnell Douglas Research Laboratories, St. Louis, MO. Since 1996, he has been a Consultant with TNO Defence, Security, and Safety, The Hague, The Netherlands. His research interests are the analytical, numerical, and physical aspects of the theory of electromagnetic waves. In particular, he is involved with efficient techniques for the computational modeling of electromagnetic fields and their application to detection and synthesis problems from several areas of electrical engineering. 